Utah State University

DigitalCommons@USU

$1-1-2013$

\title{
Scalable Sensor Management for Automated Fusion and Tactical Reconnaissance
}

Thomas J. Walls

Michael L. Wilson

Darin C. Partridge

Jonathan R. Haws

Mark D. Jensen

Troy R. Johnson

See next page for additional authors

Follow this and additional works at: https://digitalcommons.usu.edu/sdl_pubs

\section{Recommended Citation}

Walls, Thomas J.; Wilson, Michael L.; Partridge, Darin C.; Haws, Jonathan R.; Jensen, Mark D.; Johnson, Troy R.; Petersen, Brad D.; and Sullivan, Stephanie W., "Scalable Sensor Management for Automated Fusion and Tactical Reconnaissance" (2013). Space Dynamics Lab Publications. Paper 137.

https://digitalcommons.usu.edu/sdl_pubs/137

This Article is brought to you for free and open access by the Space Dynamics Lab at DigitalCommons@USU. It has been accepted for inclusion in Space Dynamics Lab Publications by an authorized administrator of DigitalCommons@USU. For more information, please contact digitalcommons@usu.edu.

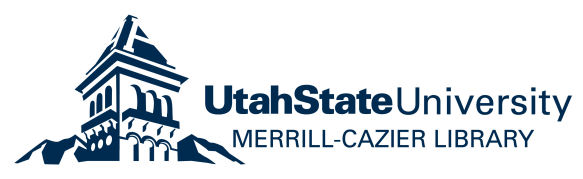




\section{Authors}

Thomas J. Walls, Michael L. Wilson, Darin C. Partridge, Jonathan R. Haws, Mark D. Jensen, Troy R. Johnson, Brad D. Petersen, and Stephanie W. Sullivan 


\title{
Scalable sensor management for automated fusion and tactical reconnaissance
}

\author{
Thomas J. Walls ${ }^{\mathrm{a}}$, Michael L. Wilson ${ }^{\mathrm{a}}$, Darin C. Partridge ${ }^{\mathrm{b}}$, Jonathan R. Haws ${ }^{\mathrm{b}}$, Mark D. Jensen ${ }^{\mathrm{b}}$, \\ Troy R. Johnson ${ }^{\mathrm{b}}$, Brad D. Petersen ${ }^{\mathrm{b}}$, Stephanie W. Sullivan ${ }^{\mathrm{b}}$ \\ ${ }^{a}$ Naval Research Laboratory, 4555 Overlook Ave SW, Washington DC 20375; ${ }^{b}$ Space Dynamics \\ Laboratory, Utah State University Research Foundation, 1695 North Research Park Way, North \\ Logan, UT, USA 84341
}

\begin{abstract}
The capabilities of tactical intelligence, surveillance, and reconnaissance (ISR) payloads are expanding from single sensor imagers to integrated systems-of-systems architectures. Increasingly, these systems-of-systems include multiple sensing modalities that can act as force multipliers for the intelligence analyst. Currently, the separate sensing modalities operate largely independent of one another, providing a selection of operating modes but not an integrated intelligence product. We describe here a Sensor Management System (SMS) designed to provide a small, compact processing unit capable of managing multiple collaborative sensor systems on-board an aircraft. Its purpose is to increase sensor cooperation and collaboration to achieve intelligent data collection and exploitation. The SMS architecture is designed to be largely sensor and data agnostic and provide flexible networked access for both data providers and data consumers. It supports pre-planned and ad-hoc missions, with provisions for on-demand tasking and updates from users connected via data links. Management of sensors and user agents takes place over standard network protocols such that any number and combination of sensors and user agents, either on the local network or connected via data link, can register with the SMS at any time during the mission. The SMS provides control over sensor data collection to handle logging and routing of data products to subscribing user agents. It also supports the addition of algorithmic data processing agents for feature/target extraction and provides for subsequent cueing from one sensor to another. The SMS architecture was designed to scale from a small UAV carrying a limited number of payloads to an aircraft carrying a large number of payloads. The SMS system is STANAG 4575 compliant as a removable memory module (RMM) and can act as a vehicle specific module (VSM) to provide STANAG 4586 compliance (level-3 interoperability) to a non-compliant sensor system. The SMS architecture will be described and results from several flight tests and simulations will be shown.
\end{abstract}

Keywords: multi-INT, data fusion, sensing modality, sensor management, network, sensor tasking, automation, algorithmic processing, scalable, systems-of-systems

\section{INTRODUCTION}

The fusion of data from multiple sources of information and sensing modalities can provide human analysts and automated detection algorithms unprecedented insight into the properties of a target or scene under examination. The combination of different scene views from across the full range of the electromagnetic spectrum provides an information rich landscape which can provide an intelligence analyst insight into target properties and intent. Examples of successful data fusion architectures span the full range of imaging, sensing and information extraction modalities. As these architectures continue to mature and demonstrate success, intelligence, surveillance, and reconnaissance (ISR) systems are increasingly being designed and built not as stand-alone sensors but as combined systems-of-systems capable of collecting data at multiple points along the information spectrum.

While the widespread proliferation of airborne sensors has provided a force multiplier for the intelligence analyst, the full potential for data fusion remains largely untapped because the time interval between data collections often proves to be too large to provide information that can be correlated about a specific target. Moreover, even sensors integrated into

Multisensor, Multisource Information Fusion: Architectures, Algorithms, and Applications 2013,

Proc. of SPIE Vol 8756, 875605 - (c) 2013 SPIE · CCC code: $0277-786 X / 13 / \$ 18$

doi: $10.1117 / 12.2018296$

Proc. of SPIE Vol. $8756875605-1$ 
a single payload typically operate in a "stovepipe" configuration, illustrated schematically in Figure 1, where the separate sensing modes operate largely independent of one another. This makes coordinated operation difficult and the generation of multi-intelligence analysis products unnecessarily slow. During a mission, it is difficult for an operator to monitor sensor data, take into account sensor field of regard at a given instant, and cue sensors to emerging areas of interest in a timely manner when each data stream is viewed independently.

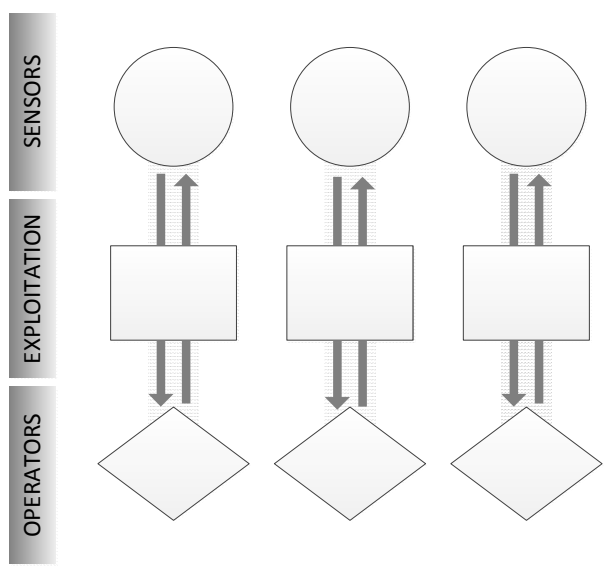

Figure 1. Common sensor data collection and exploitation architectures.

In addition, system-of-system sensor suites deployed on unmanned aerial systems (UASs) typically cannot provide data communication bandwidth capable of transmitting all of the collected data to a remote operator in real time. To achieve a true, robust, real-time data fusion capability, the sensor systems themselves need to operate in a largely autonomous, coordinated manner. This research goal is being addressed by the Office of Naval Research (ONR)-funded Tactical $\mathrm{EO} / \mathrm{IR} / \mathrm{SIGINT/SAR}$ Integrated for Targeting (TEISIT) program, which is developing an integrated suite of sensors capable of autonomous, interactive data collection based on in-scene observations and within the size, weight, and power (SWAP) restrictions of a Group III UAS. This capability allows for the collection of all relevant tactical information, upfront in the collection timeline to ensure data completeness and eliminate the need to revisit the target or scene. The system also enables the simultaneous tasking of single sensor assets between multiple users and between human and automated cueing requests to enable a single sensor asset to support multiple, simultaneous missions and/or requirements.

Central to that goal is a flexible architecture for managing the sensors that addresses the most challenging aspects of sensor autonomy and multi-sensor missions, specifically addressing the challenges of mission planning, sensor command and control, data storage, data sharing, cross-cueing, and operator tasking overload. Without a central management architecture capable of managing sensors regardless of modality or type, the system complexity increases exponentially with each new sensor added to the system. This paper describes the Sensor Management System (SMS), which has been designed and implemented to address the challenges of multi-sensor ISR missions, autonomously handling cueing and many of the mundane and routine tasks, while still allowing for dynamic mission re-tasking.

\section{SMS FUNCTIONALITY}

\subsection{Collaborative and Intelligent Data Collection}

The core of the SMS system is a small footprint, intelligent data logger that simultaneously captures, stores and redistributes data products collected by multiple sensors and produced by autonomous exploitation algorithms. The expanded system capabilities are based on software processing that understands and leverages the collected sensor data, creating a real-time awareness of the current operational situation. This situational awareness at the system core enables collaborative data collection and exploitation relevant to the current operational situation by tasking the on-board sensors to investigate scenes of interest. This facilitates a cooperative environment, prioritizing the points of interest based on predefined rules to meet both the pre-planned mission goals and dynamic mission adjustments. These dynamic sources of information can come from on-board sensors and/or be pushed to the SMS from remote sources or users. 
The SMS becomes a single coordination point for controlling payloads and obtaining sensor data, mission progress, situational awareness, and tasking accomplishments. The SMS provides a means by which multiple consumers of data are connected to the producers of data.

\subsection{Algorithms}

Running on the SMS are algorithms capable of processing multiple sources of sensor data, from which automatically generated measurement and signature intelligence (MASINT) products are produced. These MASINT products can be viewed by analysts as well as coupled to mission objectives to generate dynamic targets. When MASINT products are combined with mission objectives, preselected targets of interest, and targets added by users, the SMS algorithms generate a stream of real-time cues to task the on-board sensors.

\subsection{Modular Architecture}

The architecture of the SMS was designed to be modular, dynamic and easily adaptable. The core structure is a set of algorithms called "managers" that handle the collection of data, tasking of sensors, coordination of information exchange, operation of product generation algorithms, and execution of mission goals. These managers allow the addition of modules that extend the SMS's base functionality without any changes to the core software. Examples of such modules could include a sensor interface to control a new sensor, an algorithm that generates MASINT products, or a server that provides access to a data product or information stream. Modules can even be located at remote locations, provided they have a network connection to the SMS.

\subsection{Standards Compliance}

STANAG 4575 describes the NATO Advanced Digital Storage Interface (NADSI) standard. For data accessibility, the SMS has been developed to be a removable memory module (RMM) compliant with this standard. As a result, NADSIcompliant workstations can easily connect to the SMS system and download the raw sensor and mission data following a mission to ingest into common databases for further data dissemination.

STANAG 4586 describes UAS interoperability from a common, compliant ground station and allows for command and control (C2) of compatible sensors on-board a UAS. The SMS provides an interface that will make each sensor configured in the SMS available as a STANAG 4586 compliant sensor by way of a Vehicle Specific Module running on the SMS to perform the necessary translation and routing of information.

\section{SMS ARCHITECTURE}

The overarching purpose of the SMS is to manage and task an integrated network of sensors, collect their data, and produce synergistic data products that increase the situational awareness of the operator and tactical usefulness of the data products themselves. To accomplish this, the SMS architecture revolves around the concepts of "detections" and "cues." Detections are raw points of interest as specified by data analyzers (exploitation algorithms, processing agents, operators connected via data links, etc.) and are managed by the Data Routing and Processing Manager (DRPM) process (section 3.3). Cues are actual points of interest that a sensor is tasked to image and are managed by the Sensor Interface Manager (SIM) process (section 3.4). The SMS is composed of four major subsystems that work together to manage the collection of detections and cues. A top level diagram of the SMS is shown in Figure 2. The communication between sensors, processing agents, and the core database is coordinated through the manager structures to enable modularity between sensors and exploitation algorithms. 


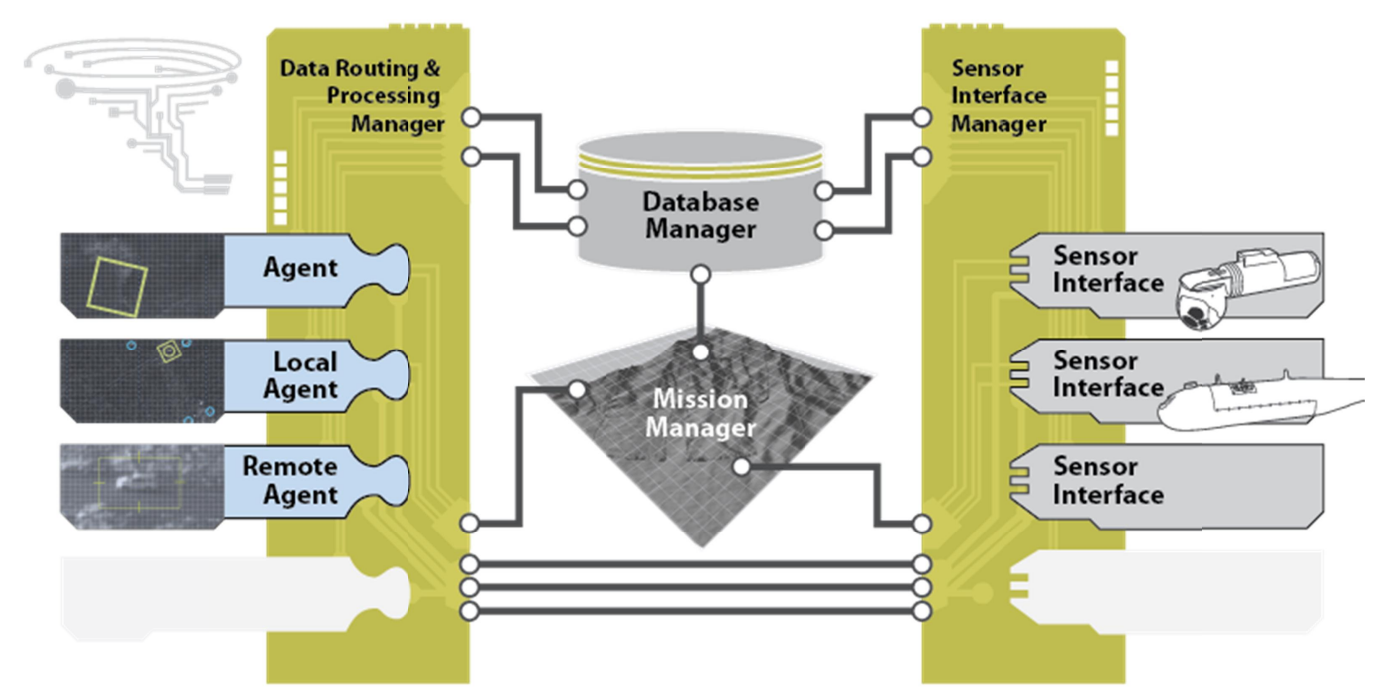

Figure 2. Top-level diagram of the SMS architecture, with modular, swappable agents and sensor interfaces.

\subsection{Mission Manager}

The SMS manages its configuration and mission plan by way of the Mission Manager (MM). The purpose of the MM is to provide a single interface for new sensor interfaces and processing agents, which register themselves with the SMS using XML strings to describe their communication parameters, rules, and other pertinent information. These registered parameters allow the SMS to properly format all future mission communications.

The MM also maintains a copy of the mission plan describing where the UAS is expected to fly. This information is fed to the SIM and DRPM for use in their real-time operations. Both of these managers are described in more detail below.

\subsection{Data Storage and Database}

The purpose of the database is to store information in an organized and retrievable fashion. On startup the database is initialized via the MM process with configuration information regarding the mission plan, sensors being managed, onboard data processing routines, system parameters, etc. The database is updated throughout the mission to represent the current state of sensors, processes, and connected users. As data is collected by sensors or data analysis algorithms and is produced by exploitation processes, it is stored to disk and addressed via the on-board database. The associated metadata and the file location on disk are maintained in the database, making any data that has been collected accessible and able to be retrieved via metadata. This capability ensures the in-flight, post-collection data is available at any time during the mission and can be retrieved and correlated against future data collections.

\subsubsection{Database Manager}

The Database Manager is a software module that maintains the SMS database described above. It serves as a single point of access for the other threaded processes running on the SMS, such as the SIM and DRPM. A module dedicated to this function is necessary to prevent conflicts and ensure the integrity of the database. The other core software modules communicate over a network connection using a defined command set. These commands allow for data to be entered in or retrieved from the database.

\subsection{Data Routing and Processing Manager}

One key challenge of the SMS is maintaining data routing integrity within the system. The objective of the DRPM is to manage data routing between data sources and data users, which are referred to as agents. The DRPM is also responsible for managing detections and their promotion to cues. The concept of agents and detections and how they are managed is described here. 
When a new processing agent comes online, one of its first tasks is to inform the data routing process of its location so that the SMS core router can push any requisite data to the agent. This data will include the type of link connection, from a high-bandwidth local connection to a low-bandwidth data link. Once the processing agent finishes this handshaking with the SMS, the SMS will return an identifier that the agent can use to identify itself to the SMS in further communications. The agent can then subscribe to any data feeds of interested. For example, a constant false alarm rate (CFAR) detection algorithm may only be interested in appropriate radar data, while an off-board screener may request access to all relevant imagery data and detections. The DRPM exposes each agent only to those data streams relevant for its mission objectives.

\subsubsection{Agents}

"Agent" is a term used to describe any software module that connects to the SMS for the purpose of receiving raw or processed data, producing processed data, and/or tasking sensors. Agents can be an interactive software application where a user views data and sends commands directly to the SMS or they can be algorithms that run autonomously on raw sensor data to extract information pertinent to the mission. Agents communicate with the SMS via IP sockets and can be plugged into and removed from the SMS at any point in time.

\subsubsection{Agent Manager}

The Agent Manager (AM) is the process that handles all the data routing to agents, as well as managing the process of elevating reported detections to sensor cues. The data routing and rules for elevation are dynamically maintained, such that as agents and sensors enter and leave the network, routing tables and elevation rules are updated to maximize efficiency of operation.

\subsubsection{Data Routing}

After an agent has registered with the SMS and communication parameters have been established, the agent will typically subscribe to some data feeds. To enable this, the SMS maintains a list of data feeds that have been registered by sensors or other agents. For an agent to request real-time data, it can subscribe to the registered data feed by name. This act of subscription establishes a link between the DRPM and the agent for the purpose of getting data to the agent. As metadata is updated in the database, the DRPM is notified of any subscribers to that data. If an agent has subscribed to the data feed, the data is pulled from the file and transmitted to the subscriber via the previously established socket.

\subsubsection{Detection Management}

Another key task of the DRPM is to determine if there is a configured sensor interested in a received target or detection. If such a sensor exists, the received detection is elevated to a cue based on the rules and capabilities of that specific sensor and is stored in the cue list for that sensor.

\subsubsection{Detection Rules}

A detection arriving at the SMS is immediately stored in the database, after which a cue transition module is responsible for promoting detections to cues. The flow of detections entering the SMS and being promoted to cues is illustrated in the following figure. This promotion is based on rules that were established in the sensor configuration. The main SMS XML configuration file contains a list of detection sources. If a detection source is listed in a sensor's configuration, detections from those sources will be promoted as cues for the corresponding sensor. This allows for multiple sensors to accept cues from a single source; thus, should two sensor configurations specify detection rules for the same source, two cues will be created when those detections are promoted to cues - one for each sensor. 


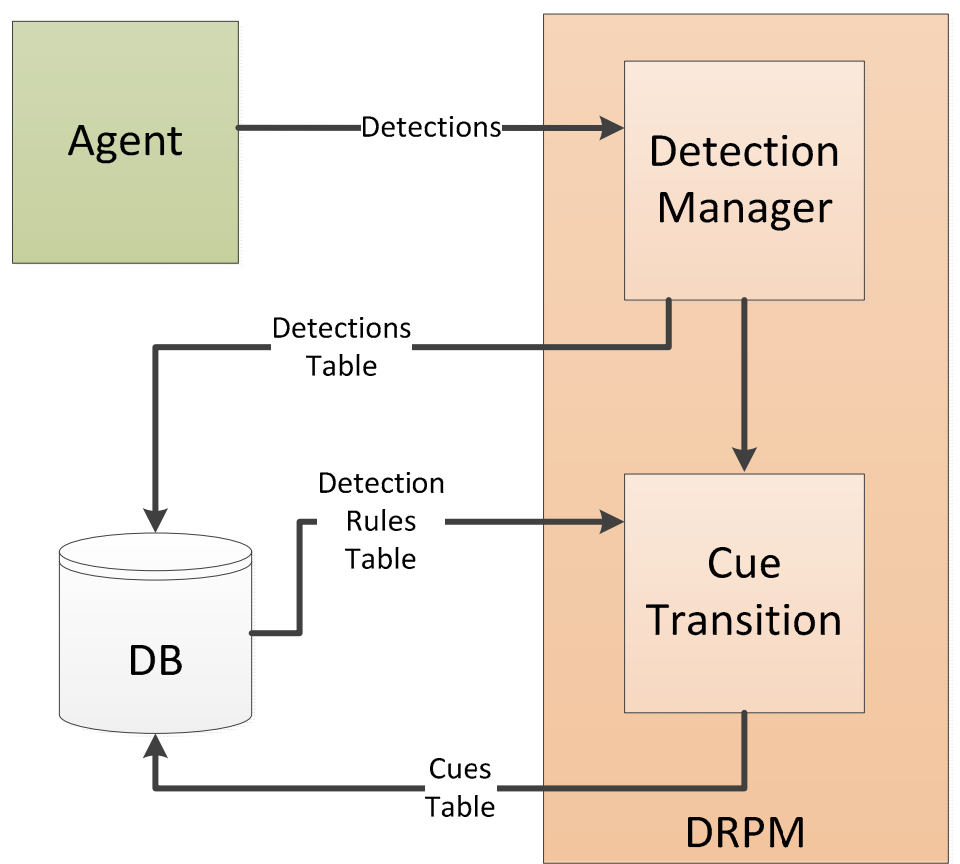

Figure 3. Detections from agents are fed into the detection manager and immediately added to the database. The cue transition block compares the detection source to the list of rules in the database to determine if this detections needs to be sent to a sensor. If a sensor is found, the detection is elevated to a cue and written back to the database as a cue.

\subsubsection{Agent Filtering}

One of the powerful features of the SMS is agent filtering. Agent code can be connected to the SMS, which receives detections or data from other agents and sensors, fuses this information together and, by doing so, is able to create new, more intelligent detections. Fused data products and advanced decision making algorithms can then be assigned the task of generating cues for a given sensor or modality.

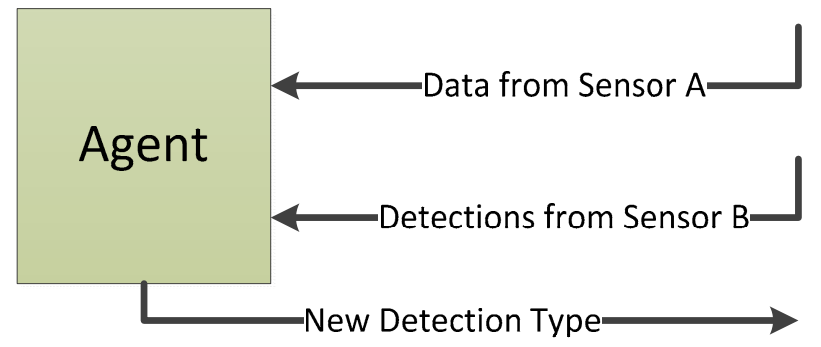

Figure 4. An agent can be created to receive sensor data and detections in order to correlate existing detections against the new data. This will produce a more intelligent detection source.

A simple example of this might be the following. An agent could be created that receives Automatic Identification System (AIS) detections and synthetic aperture radar (SAR) detections. It then produces detections of bright objects in the SAR images that do not have a corresponding AIS detection. In this way, the imaging sensor could be cued to look at only bright SAR objects that have not been already identified by AIS detections or whose size and shape characteristics do not match the information reported by the AIS signal.

\subsubsection{Detection Flags}

Each detection can be characterized by a set of flags, described in Table 1. These flags help signify the unique properties for detections that require special handling. For example, detections that require periodic revisiting or target detections that are moving require time sensitive sensor cueing. Under normal operations, once a detection has been cued and 
imaged by a sensor, the detection/cue is marked as completed and is not reimaged unless a subsequent detection is sent or one of the flags listed in Table 1 is specified when the detection structure was sent.

Table 1. Detection flags classify detections further than just their source.

\begin{tabular}{|l|l|}
\hline \multicolumn{1}{|c|}{ NAME } & \multicolumn{1}{c|}{ DESCRIPTION } \\
\hline Persistent & $\begin{array}{l}\text { Detections flagged as persistent are never marked as completed. They will be } \\
\text { continually imaged until the detection is deactivated. }\end{array}$ \\
\hline Moving & $\begin{array}{l}\text { Detections flagged as moving will be considered volatile and multiple } \\
\text { detections of this type with the same ID will be used to calculate a current } \\
\text { position based on a calculated projection. }\end{array}$ \\
\hline User & $\begin{array}{l}\text { Identifies the origin of the detection as a human. Allows weighting algorithms } \\
\text { to favor detections created by users. }\end{array}$ \\
\hline Timeout & $\begin{array}{l}\text { Indicates the Timer Seconds field in the SMS Detection structure is a period } \\
\text { over which the detection is valid. Once the amount of time specified has } \\
\text { elapsed, the detection/cue will be marked as inactive. }\end{array}$ \\
\hline Time Interval & $\begin{array}{l}\text { Indicates the Timer Seconds field in the SMS Detection structure is an interval } \\
\text { for which the detection should be imaged. }\end{array}$ \\
\hline Best Score & $\begin{array}{l}\text { Indicates that if a better score for a cue becomes available, the SMS should } \\
\text { consider targeting the detection again. }\end{array}$ \\
\hline
\end{tabular}

\subsection{Sensor Interface Management}

The assumption made by the SMS architecture is that each sensor connected to the system communicates using its own proprietary format for $\mathrm{C} 2$. This presents a challenge in providing $\mathrm{C} 2$ level access to a common system. The SMS addresses this challenge by abstracting the sensor interfaces away from the core sensor control code. In this way, sensor management is broken up into two pieces - the SIM and sensor interfaces, which are sensor specific.

\subsubsection{Sensor Interface Manager}

The SIM indirectly sets up and controls all sensors attached to the SMS. A sensor is attached to the SMS through a sensor interface that is specific to each sensor and sensor class. The sensor interface is a stand-alone module that contains the methods for translating the sensor's data product into a standard format understood by the SMS and provides a set of commands allowing the SMS to control the sensor. The SIM communicates and controls the sensor through this interface module.

The SIM controls the intelligent collection of data by coordinating the tasking and cueing of the sensors. The SIM utilizes prioritized, filtered cues based on a configurable set of rules to determine where a sensor should be tasked. Multiple sensors can be simultaneously tasked to image the same location to provide input for data-fusion MASINT products.

\subsubsection{Cue Management}

The key role for the SIM is to manage the cues that are sent to sensors. This management takes place in several steps. These steps can be seen in the various blocks found in the SIM prioritization diagram shown in Figure 5. 


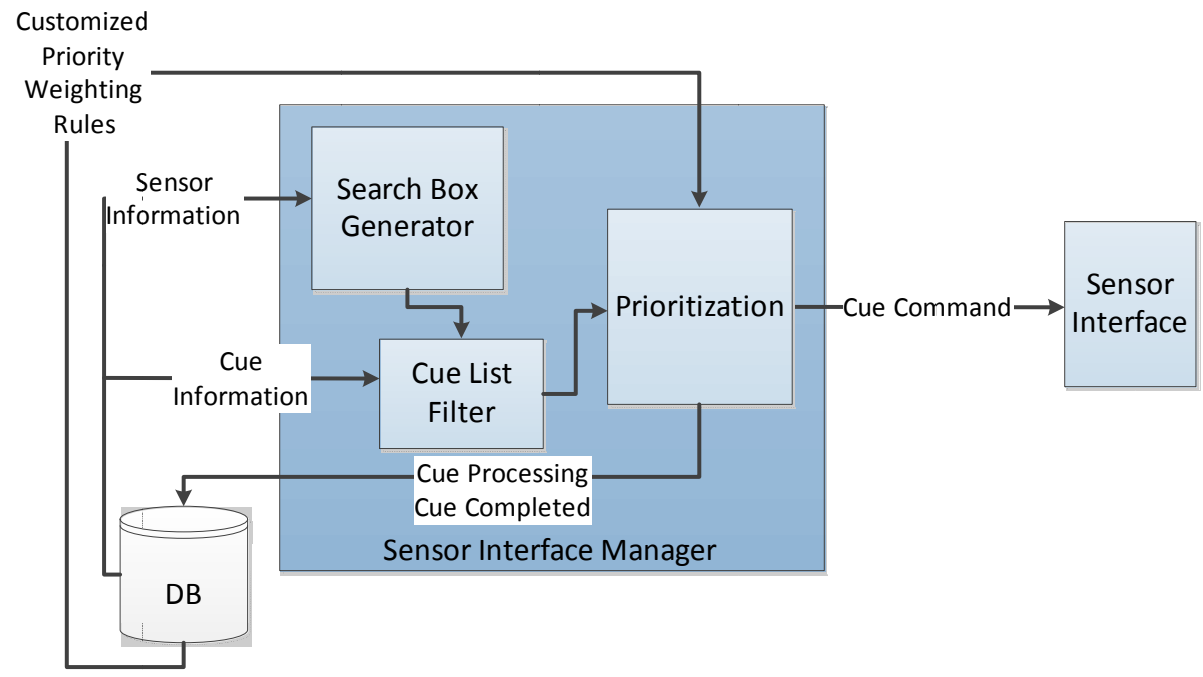

Figure 5. The SIM prioritizes and filters cues down to the best cue to be sent to the sensor.

Before a cue is sent to a sensor, the number of cues is narrowed down by filtering and prioritization so that the best cue is selected. Each of these steps is covered in the following sections.

\subsubsection{Geographic Filtering}

Cue management begins by filtering all the cues available to a sensor, down to those that are actually within the geographic field of view (FOV) of the sensor. This is done by using a search box that is defined and configured when a sensor undergoes its initial setup and handshaking with the SMS. A search box is defined to be an area in which a sensor is capable of investigating or imaging, and is defined to account for the real-time motion of the imaging platform. It is configured by specifying a FOV using four parameters: azimuth size (degrees in the cross-track direction), elevation size (degrees in the flight path direction), azimuth offset (degrees), and elevation offset (degrees). The azimuth and elevation sizes set the size of the search window, while the azimuth and elevation offsets set the position of the search window. If the offset values are both zero, the window is centered directly below the platform. A positive elevation offset moves the search window forward and a negative offset moves the window backward relative to the position of the aircraft. A positive azimuth offset moves the search window to the left of the plane and a negative offset moves the window to the right of the plane. In Figure 6, the azimuth offset is positive and the elevation is negative.

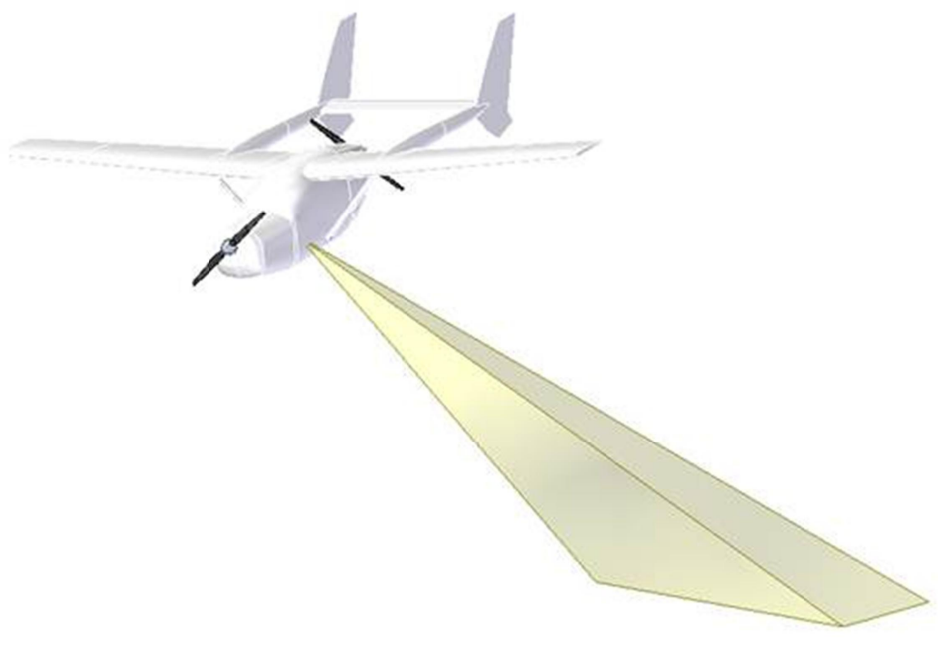

Figure 6. The search box shown here has a positive azimuth offset, which moves the search box to the left of the airplane. The negative elevation offset moves the search box behind the airplane. 
The SMS only sends cues to sensors when a cue falls within a sensor's search box. In this way the SMS is able to limit the targeting of detections to intervals in which the sensor will be able to best observe or image the detection. These values should be set up with this goal in mind.

\subsubsection{Cue-level Filtering}

Cue-level filtering ensures that the cue is actually viewable from the current vantage point and is not obstructed by objects or terrain. Terrain, ground sample distance (GSD) and other filtering modules can be added to the SMS, which will use these filters to further reduce target cues to just the set for which the sensor can provide useful information. For example, flight planning software could create areas in which detections should or should not be targeted.

\subsubsection{Scoring}

Every cue inside of the search box is given a score based on the specified weighting rules customized in the sensor configuration. The cue with the highest score is used to generate the next imaging command to the corresponding sensor. Each sensor is set up with a unique scoring algorithm by adding rules, or weighting functions, to the sensor configuration settings, and can be configured with independent sets of rules and weighting functions. The weighting factors used to determine a cue's score are shown in Table 2.

With each weighting function, a value from $[0,1)$ is used to weigh how heavily this function factors into the overall score. These can be thought of as ratios. If one weighting function was given the value of 0.3 and a second function has a value of 0.9 , the score generated would reflect a value with the second function weighted three times more heavily. A weighting function can also be completely disabled by simply weighing it with a value of zero in the sensor configuration.

If all of the weights are equal, then each function would be considered equally in scoring. There would be no difference in the relative scores if every function was weighted with the value of 0.1 or if every function was weighted with the value of 0.9. For this reason, it is important to be judicious during sensor configuration to ensure that a valid scoring function will be used to prioritize cues based on mission objectives. For example, one mission's objective may be to ensure that the best images of targets are taken; thus an electro-optical/infrared (EO/IR) sensor would ensure that it weighs distance and slant angle more than the others. However, a different mission may want to ensure that anything that is moving in the scene is imaged immediately; thus an EO/IR sensor would ensure that the moving and time functions have higher weights than the other functions.

Table 2. Weighting functions that are currently supported by the SMS

\begin{tabular}{|l|l|}
\hline \multicolumn{1}{|c|}{ NAME } & \multicolumn{1}{c|}{ DESCRIPTION } \\
\hline Distance & $\begin{array}{l}\text { The distance between the detection and the current location of the platform. } \\
\text { The closer a detection is to the platform the higher the score. }\end{array}$ \\
\hline Time & $\begin{array}{l}\text { The time since the detection was added to the SMS's database. The newest } \\
\text { detections will have the highest score. }\end{array}$ \\
\hline Slant Angle & $\begin{array}{l}\text { The angle of the slant range from the platform to the detection. The smallest } \\
\text { slant angle will have the highest score. }\end{array}$ \\
\hline Moving & $\begin{array}{l}\text { When this weighting function is used, a detection that has been flagged as } \\
\text { moving will be given a score greater than a detection that is not moving. }\end{array}$ \\
\hline User Priority & Integrates the priority assigned by a user into the score. \\
\hline Agent Priority & $\begin{array}{l}\text { Integrates the priority of an agent (normally assigned to the agent in the SMS } \\
\text { configuration) into the score. }\end{array}$ \\
\hline Detection Priority & Integrates the priority assigned by an agent into the score. \\
\hline Altitude & $\begin{array}{l}\text { Utilizes above ground level (AGL). Detections with the smallest AGL will } \\
\text { have the highest score. }\end{array}$ \\
\hline Time Critical & $\begin{array}{l}\text { When this weighting function is used, a detection that has been flagged as time } \\
\text { critical will be given a score greater than a detection that is not. }\end{array}$ \\
\hline
\end{tabular}




\subsubsection{Sensor Interfaces}

Sensor interfaces are the means by which the SMS communicates with each sensor attached to the system. They act as translators between the sensor protocol and the SMS protocol. This adds a layer of abstraction over the sensor (see Figure 7), making it possible for to the SMS to communicate with any sensor via a common sensor interface, rather than using the sensor's native protocol. This grants a high level of flexibility to interfacing with new sensors.

Sensor interfaces are similar to agents in that they connect to the SMS via IP sockets. Using this connection, sensor metadata and data are passed to the SIM for archival in the SMS database. Because sensor interfaces communicate via standard sockets, they can reside anywhere on the sensor network rather than being required to run on the same hardware as the SIM. This allows for a sensor to be located on the ground or even on another aircraft, as long as a communication path between the interface and the SMS exists.

Another benefit of the socket-based architecture is that it allows for sensors to come and go on the sensor network. In order to establish communication with the SMS, a sensor interface will register its sensor with the SMS, thus plugging itself into the SMS network. In the same fashion, a sensor interface can leave the network and the SIM will dynamically adjust its priorities and cueing tables to account for the disconnected sensor.

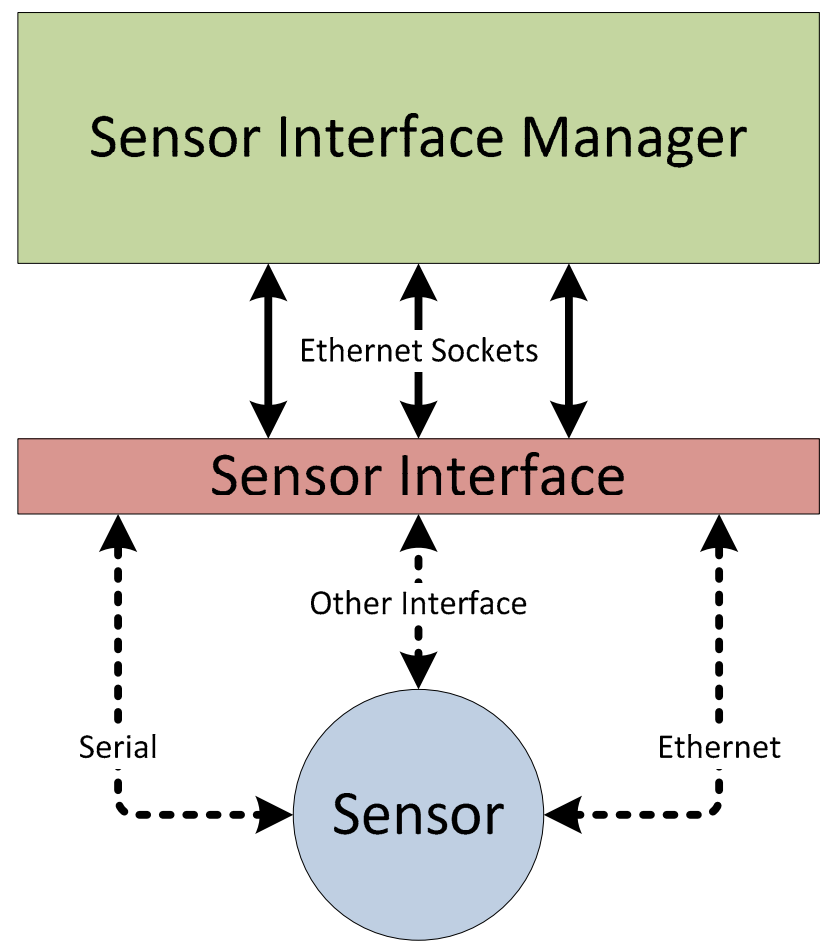

Figure 7. The sensor interface acts as a layer of abstraction from the sensor itself.

\section{IMPLEMENTATION AND TESTING}

The core SMS modules have been written and flight tested in a portable $\mathrm{C}$ implementation, compiled for the Linux operating system. This includes all of the manager implementations described above as well as sensor interface modules for the EyePOD EO/IR and Real-time Autonomous Synthetic Aperture Radar (RASAR) sensors [1,3] and a commercial off-the-shelf (COTS) AIS sensor. Processing agents for the EarthView screener [3] and a CFAR real-time target detection for the SAR imagery produced by the RASAR sensor have also been implemented and demonstrated in live 
flight testing. The current implementation of the SMS software is running on an embedded form factor system. This small, embedded implementation was designed to meet the SWAP goals of the TEISIT program.

Functional testing of the SMS system was accomplished by live flight testing the SMS with attached sensors and via a mission simulation capability. In both scenarios, complete sensor data logs were stored for later inspection to ensure correct mission operation, and the quality and reliability of MASINT products generated by the agents were assessed. The speed and efficiency at which the SMS can ingest MASINT products and task the sensors based on this data were measured. A variety of SMS agents pulled mission data, both in real-time and post flight, to test these connections.

Preliminary testing of the SMS was performed with simulated data. The mission simulation capability enables the evaluation of the current state of each sensor's cue list to determine how each sensor is responding to various detection input lists and priorities and to exercise artificial scenarios to stress each sensor's capability to prosecute a given target list. A graphical user interface (GUI) to generate target sets was created in MATLAB ${ }^{\circledR}$. Targets can be placed manually or randomly, within a set of specified parameters. The simulated target sets can be fed into the SMS alone or along with data recorded on a previous flight. Both the simulated and actual data can be fed in at the beginning of a simulation or during run time. Flight paths can be simulated or can be recorded on an actual flight and reused in simulations. The flight GPS data can be fed into both the SMS and the sensors to simulate flight conditions.

Testing of the SMS was monitored using an SMS agent created in MATLAB. This agent connects to the SMS and records the data feeds selected by the user. Data is displayed in real-time in an interactive GUI. The GUI has the ability to send detections to the SMS in real time. The detections can be adjusted by the user to mimic detections sent by any agent. The GUI can be used during both lab and flight tests.

The majority of testing was performed by simulated flying of the SMS, along a previously flown trajectory, through simulated or pre-recorded scenarios. If using a pre-recorded scenario, exact inputs to the SMS, including GPS readings and actual sensor collections were re-sent. Additional simulated sensor collections, detections and GPS readings were sent along with the pre-recorded data to stress the SMS. Many flight tests over ground-based targets were conducted to validate simulation results.

\subsection{Simulation Setup}

In order to validate and examine the effectiveness of the cue prioritization and weighting functions extensive simulations were created and executed. Figure 9 shows the simulation setup and flow of information through the SMS.

The simulation progresses as follows:

(1) Simulated INS data is loaded into the RASAR simulation controls.

(2) Moving targets are generated using the moving target generator MATLAB tool.

(3) Static targets are generated using the "world" simulator MATLAB tool.

(4) INS data is sent through the RASAR sensor into the SMS. A message containing this data is also fed to the EyePOD simulator to ensure that both sensors are operating in the same location and on the same simulated airplane.

(5) As INS data is received by the SMS, the GPS/INS agent receives this data to produce a data feed that is available to all clients of the SMS (agents and sensor interfaces).

(6) While the airplane is flying, the simulation agent takes the generated target list and sends the targets to the SMS at varying intervals. At the same time as the RASAR sensor produces SAR imagery, the SAR CFAR algorithm scans the images and produces detections.

(7) Utilizing the rules configured in the sensor configuration, the detection to cue promotion algorithm promotes the detections received into cues for EyePOD.

(8) As the aircraft flies its simulated trajectory, the Sensor Manager is searching in the search box to see if any cues are available for the EyePOD sensor. This generates a list of cues that are sent to the prioritization block.

(9) The prioritization block receives the list of cues and prioritizes them based on the list of weighting functions received from the sensor configuration. The cue with the highest score is sent to the sensor interface for imaging. 
(10) The EyePOD sensor interface receives the cue from the Sensor Manager, images the target, and returns the images to the SMS for cataloging.

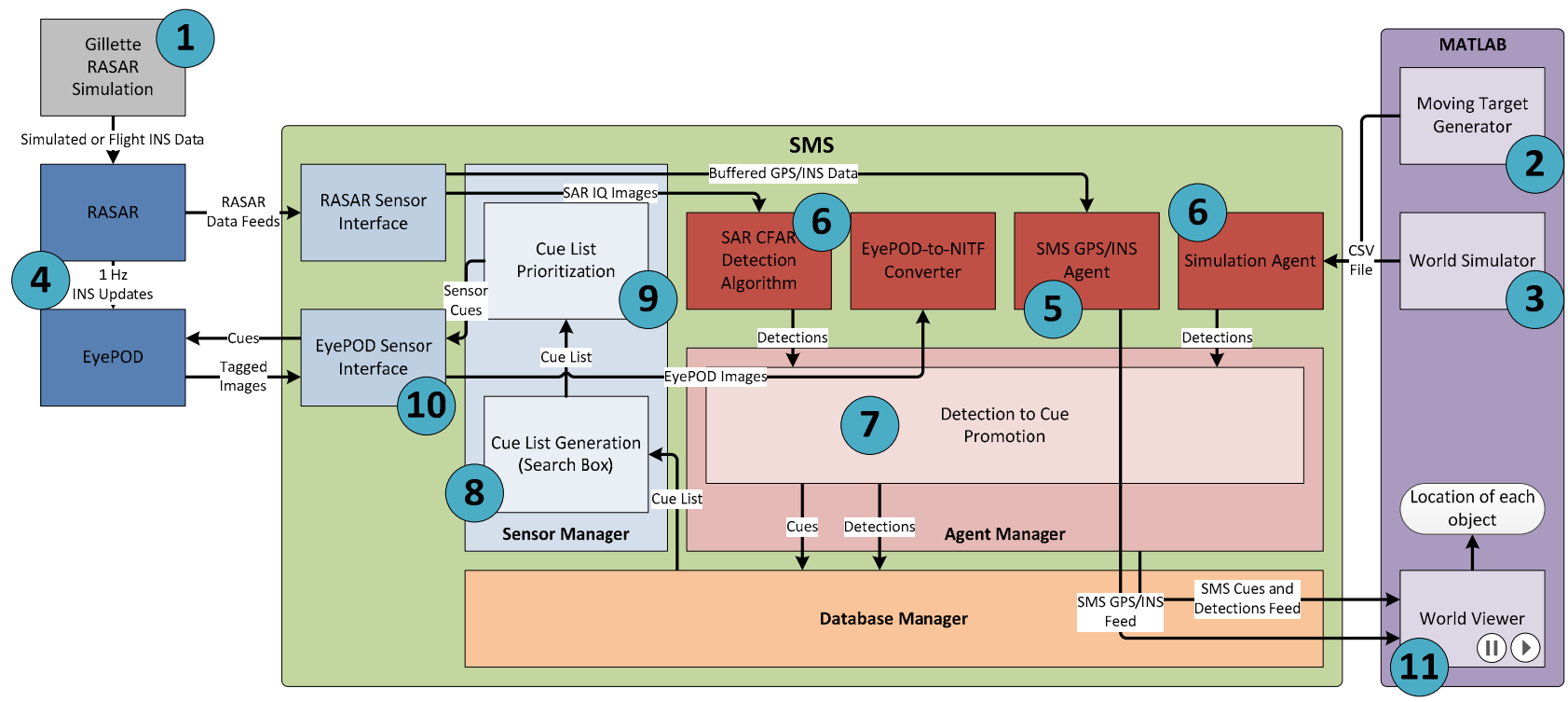

Figure 8 . The simulation setup for validating the functionality of the SMS cue management is shown.

\subsection{Simulation Scenario}

We highlight here one simulated testing scenario designed to exercise the target detection and cue prioritization algorithms of the SMS. In this scenario, the airplane is orbiting around a geographic point and the actual GPS flight profile of the aircraft was captured. This flight profile is then replayed through the mission simulation capability and synthetic targets are injected into the scene. The SMS is configured to control a SAR sensor and an EO/IR sensor. Screenshots of the scenario are shown in Figure 10Error! Reference source not found.. In each figure, the legend shown in Figure 10 applies. When active targets are within the search box, the target identification number and the current score are displayed.

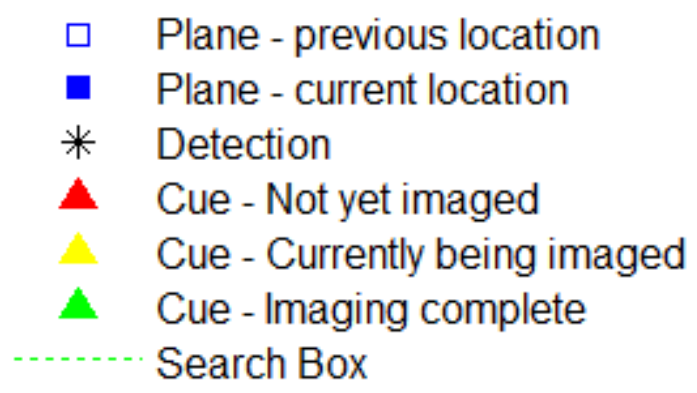

Figure 9. Legend describing the objects seen in the simulation figures.

The simulation agent reads in the pre-generated target file and pushes persistent targets to the SMS immediately. These targets are imaged by the EO/IR sensor as they become visible in the search box, as shown in Figure 11. Each target will only be imaged once, unless the target has been identified as needing repeat collections. Persistent targets, such as cue $\{10\}$ in Figure 11 and Figure 12 are imaged as defined. The simulation agent also reads in a set of moving targets and sends out the latest location of the moving target at $2 \mathrm{~Hz}$. In the same manner as persistent targets, moving targets are imaged as they are seen in the search box; the main difference is that as a mover's location is sent to the SMS as a detection, the cue representing the new location of the target is updated instead of a new one being created. This enables the sensor imaging the target to image the latest location of the moving target, rather than the entire track as shown in Figure 13. 
For this simulation, GSD considerations were valued higher than other weighting functions. This means that the weighting functions for EyePOD were set as follows:

- $\quad$ Slant Range: 0.7

- Distance: 0.5

Using these weights, the sensor manager was able to prioritize multiple cues in the search box appropriately. Based on GSD considerations, both cues $\{13\}$ and $\{18\}$ have good scores. However, though $\{13\}$ is closer to the aircraft (0.02361), its normalized slant range (0.36028) is slightly worse than the slant range of $\{18\}$ (0.38369), which lies at a normalized distance of 0.01931 from the aircraft. Applying the weights to these values results in cue $\{18\}$ having a higher score; thus it is imaged first, as shown in Figure 14Error! Reference source not found.. Once cue $\{18\}$ has been imaged by the sensor, cue $\{13\}$ will be sent to the sensor.

In the same way that the simulation agent can push detections into the SMS to cue sensors, operators also have this ability. As the SMS is flying its course, an operator connected to the system via the EarthView screener pushes a target near the center of the circle for EyePOD to image. An example of this in action is shown in Figure 15.

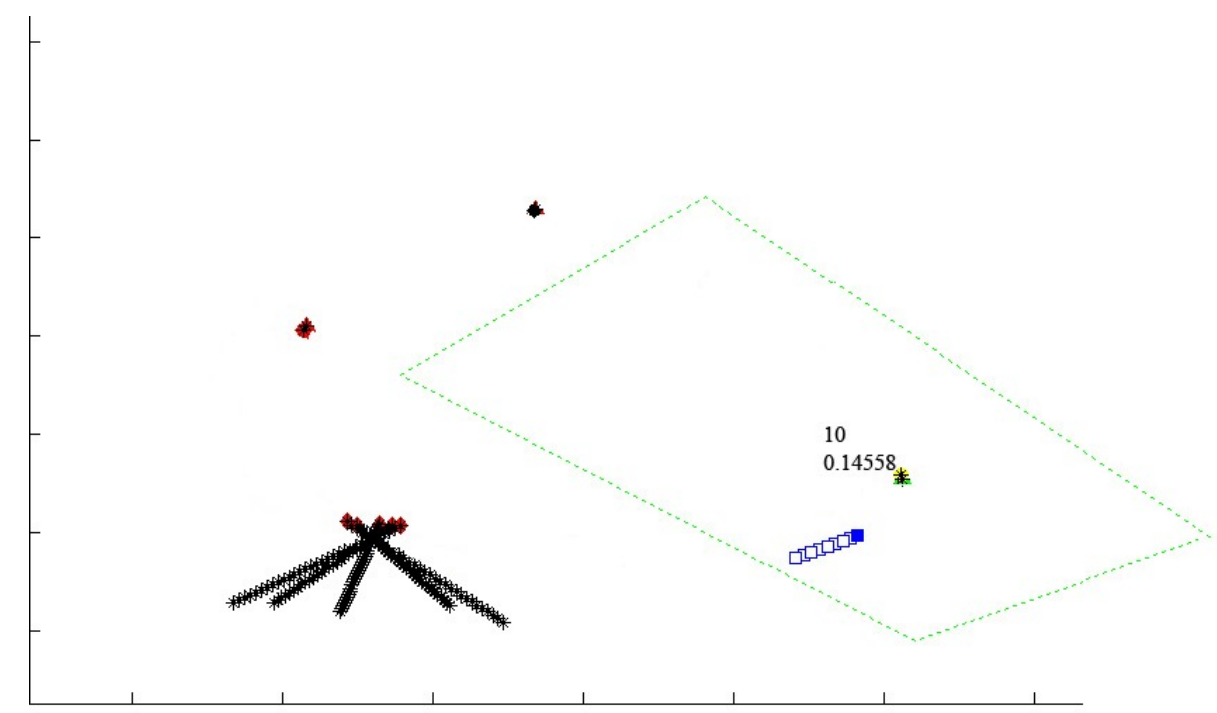

Figure 10. A persistent target is imaged when it comes into the field of view of the imaging sensor. This shows that cue $\{10\}$, with a score of 0.14558 , was imaged on this pass of the aircraft. 


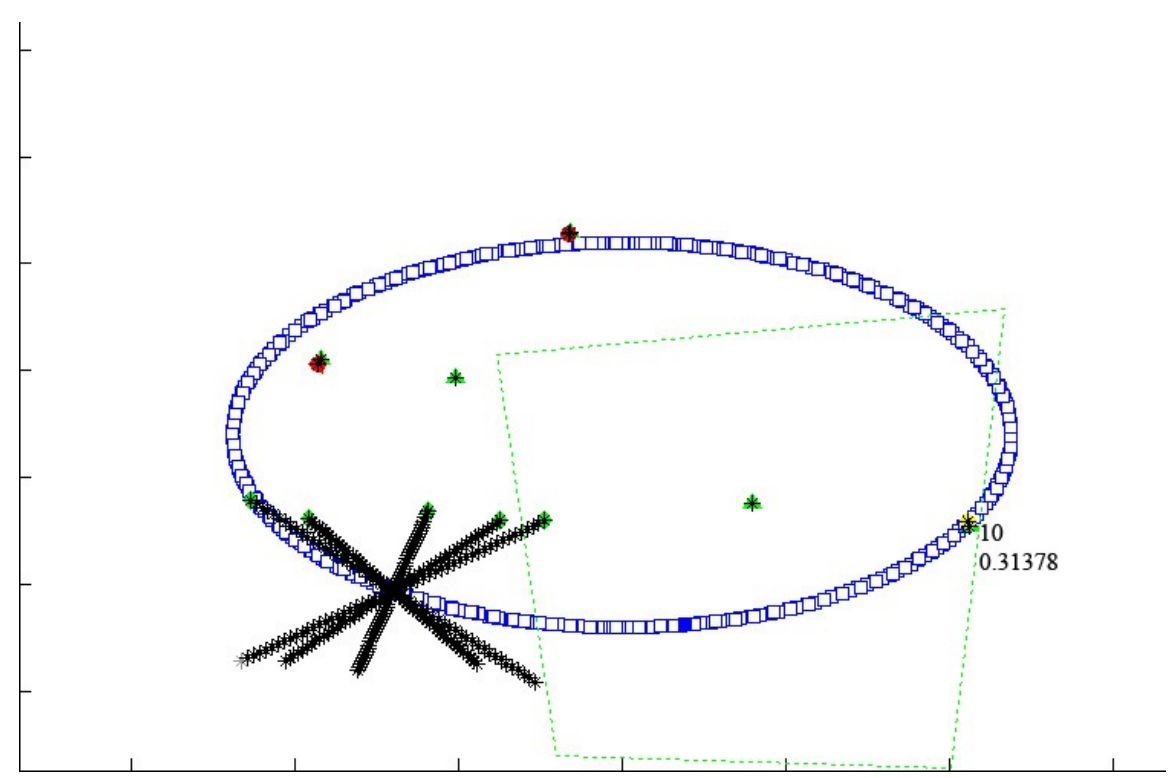

Figure 11. Because cue $\{10\}$ was a persistent target, it is imaged on each pass of the airplane.

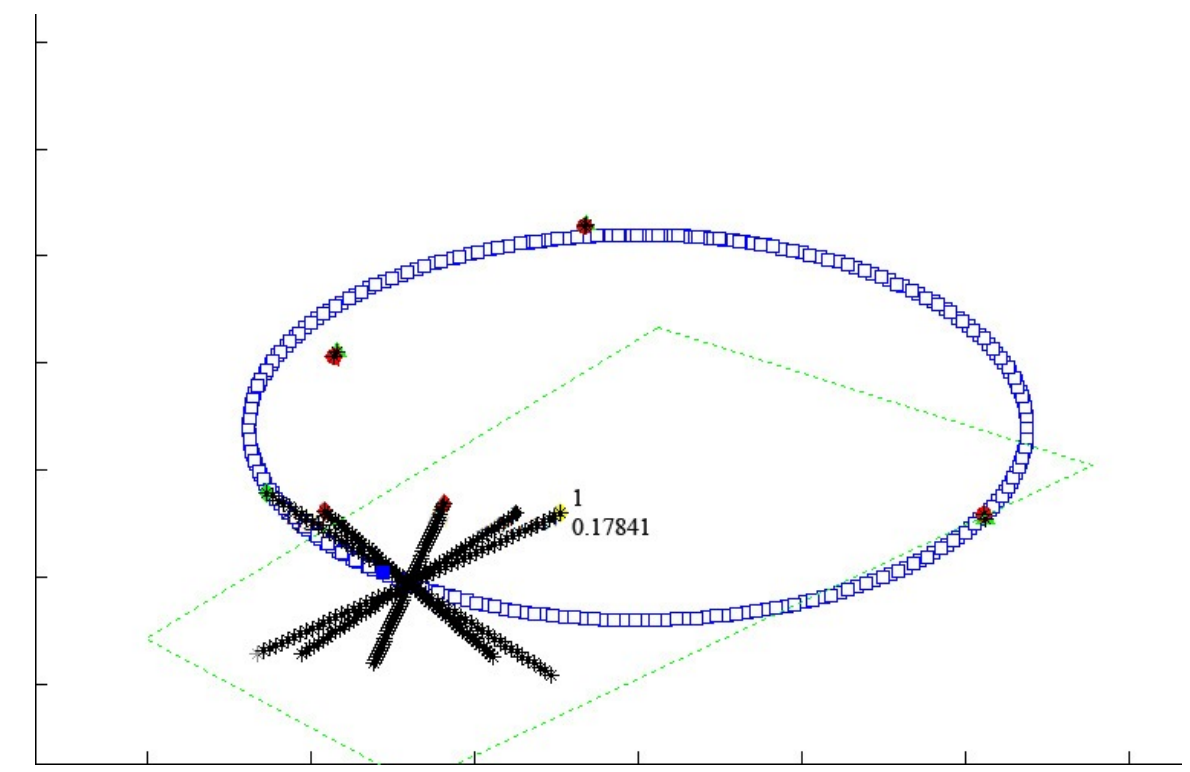

Figure 12. A moving target has its cue updated, rather than a new one created. The yellow dot at the end of the moving target's track in this figure shows that it is the point being imaged, rather than the entire track. 


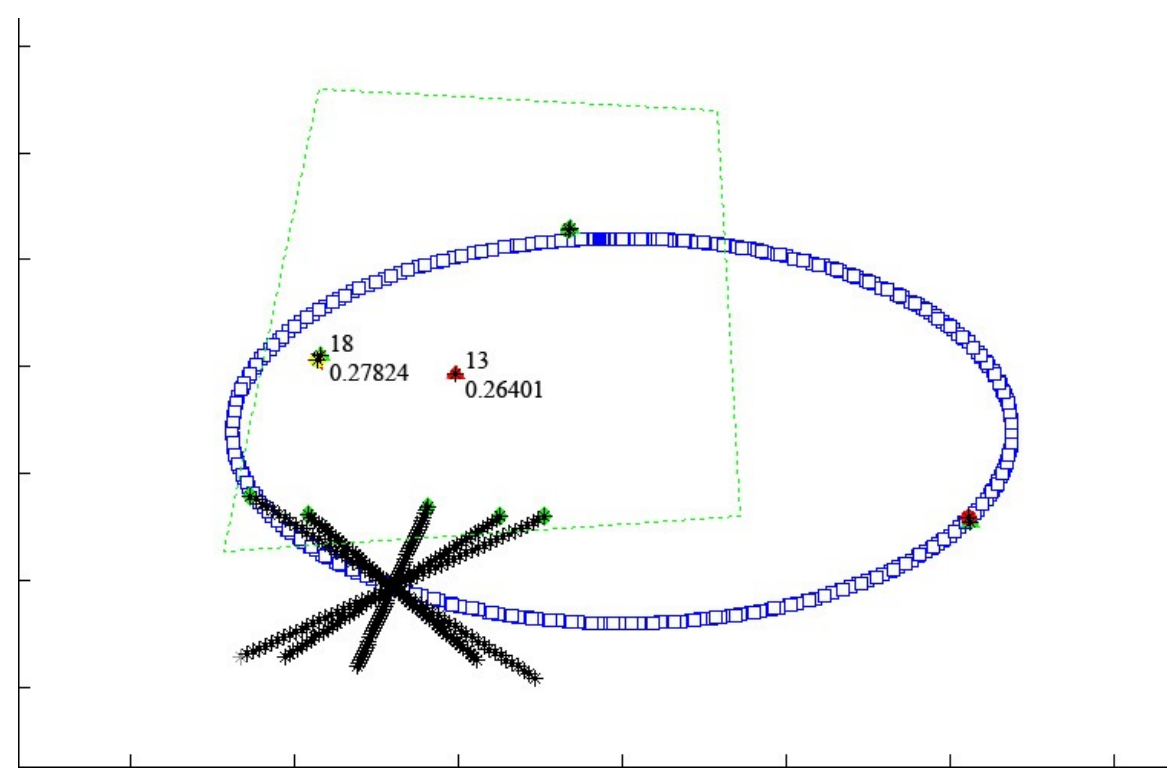

Figure 13. With the appropriate weighting functions, cue $\{18\}$ has a slightly higher score than $\{13\}$; thus is cued before it.

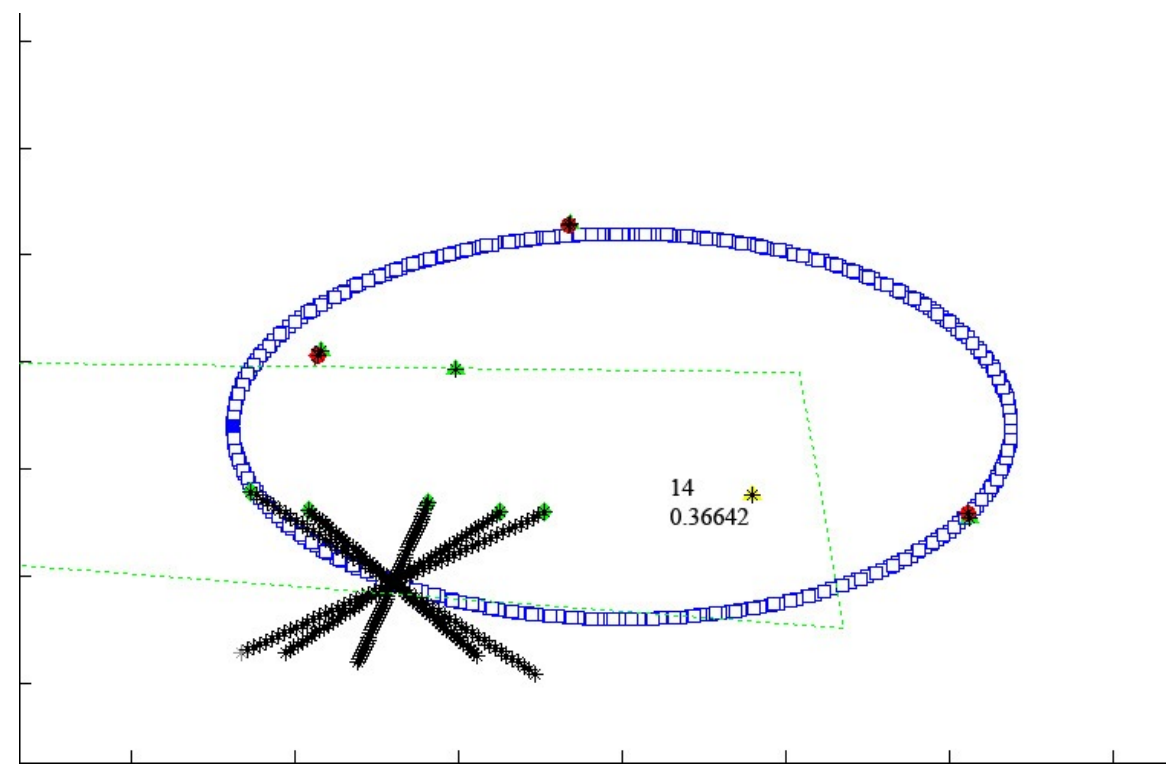

Figure 14. An operator inserted a target into the SMS queue as the airplane was flying its simulated circle. As with other cues, this cue was imaged when it had a higher score than all the others in the search box.

As shown in the simulation results, setting appropriate weighting functions for each sensor based on mission objectives is critical. Well-defined goals will determine how to weight each aspect of the detections to ensure that cues are prioritized appropriately. 


\subsection{Flight Testing}

In addition to the simulated target exercises above, the SMS system has also been demonstrated in several live flight scenarios. All flight test data shown below was collected with the UAS SWAP compatible sensors and SMS hardware installed on a surrogate, SDL-owned and operated Skymaster O-2 aircraft. Two sets of results are presented here.

\subsubsection{AIS Signals Cueing EyePOD EO/IR Sensor}

In the first scenario, the SMS system is coordinating data from an AIS receiver with the EyePOD EO/IR sensor [1]. An AIS transmitter was installed in a car driving through Cache Valley, UT, with the Skymaster aircraft in a surveillance pattern overhead. As AIS signals were received by the receiver, these were passed as detections to the SMS. The EyePOD sensor was configured to image cues generated from AIS detections. An operator on-board the aircraft was connected to the SMS and able to monitor the autonomous images in real-time. In the SIM, the received AIS detections were filtered by the search box; thus if EyePOD could not "see" the target area, the cue was not sent. This filtered cue approach demonstrated the benefit of data reduction enabled by the SMS, limiting the amount of real-time information that needed to be analyzed by the operator by excluding very oblique angles of an area that did not show what generated the cue.

This test validated the SMS performance against cueing imaging requests against moving targets. As the AIS signals were received, the detections were flagging with the "Moving" flag, described above and each target was assigned an identifier to uniquely identify the vehicle. The EyePOD sensor was successfully able to image the latest position of the target without wasting imaging resources on stale locations.

Figure 16 shows visible and infrared images from this test. EyePOD imaged the car in the top of the frame based on the cue information it received from the SMS without a human in the loop.
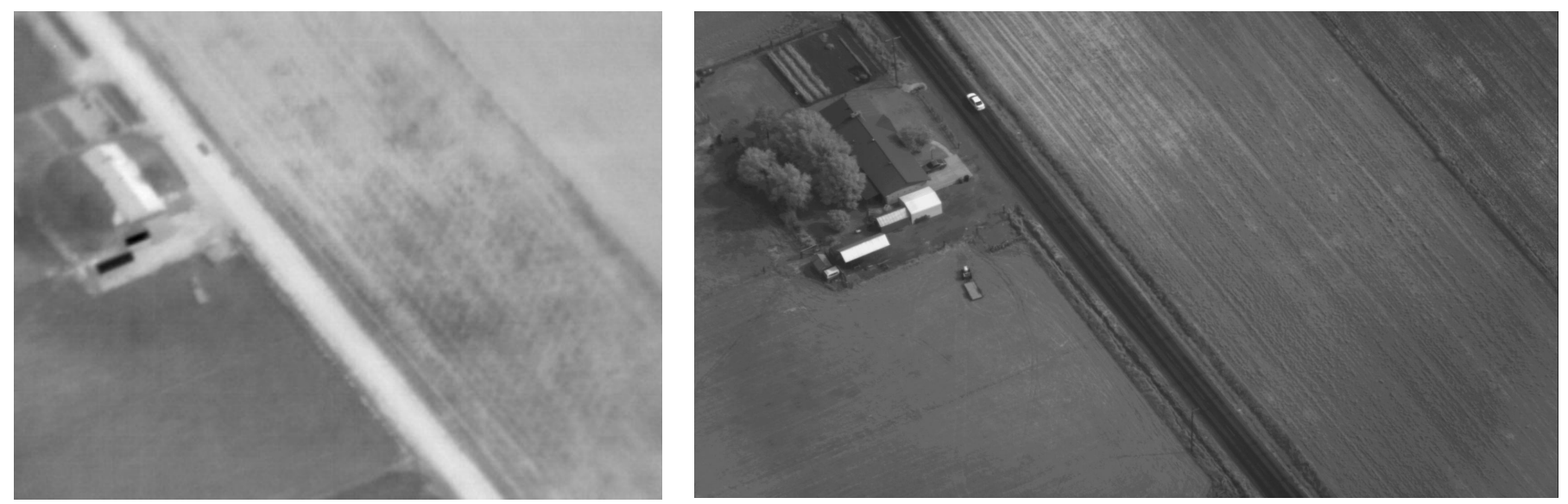

Figure 15. Infrared (left) and visible imagery (right) of vehicle carrying AIS transmitter. The AIS transmitter sent the location of the vehicle to the receiver on the airplane, which sent the detection to the SMS. The SMS then cued the EO/IR sensor to generate this imagery.

\subsubsection{RASAR Auto-detections Cueing EyePOD EO/IR Sensor}

In the second scenario, the RASAR [3] sensor was coupled with the EyePOD sensor. The SAR sensor collected data and real-time image products were generated on-board the aircraft. Utilizing processing modules within the SMS, the SAR imagery were scanned using a CFAR algorithm to generate possible targets of interest. Based on the CFAR score, these points of interest (or detections) were sent autonomously to the EyePOD sensor for further investigation. The SMS handled the passing of SAR images to the CFAR algorithm as well as the reception and forwarding of target information to the EO/IR sensor, all autonomously.

This test validated and demonstrated autonomous agents generating real-time products and detections based on sensor data, sensors receiving multiple sources of detections, valid search box filtering, and prioritization of cues when multiple cues fall inside the search box. 
Multiple targets were placed in the flight test area at the Utah Test and Training Range (UTTR) for the SAR sensor to image. The CFAR detection algorithm generated points of interest within the image, which were then translated to geolocations on the ground. These geo-locations were fed from the processing agent to the SMS, which elevated them to cues based on the EyePOD configuration rules loaded into the MM. The search box configured for EyePOD picked up multiple detections, which were then prioritized based on distance to the target and CFAR score (the detection score in Table 2). These cues were then imaged by the EyePOD sensor and correlated against the SAR detections, providing the operator or analyst not just SAR points of interest, but also high resolution optical imagery of the target for identification. This autonomous imaging chain of events is shown in Figure 17, with the correlated optical image of the target vehicle highlighted.

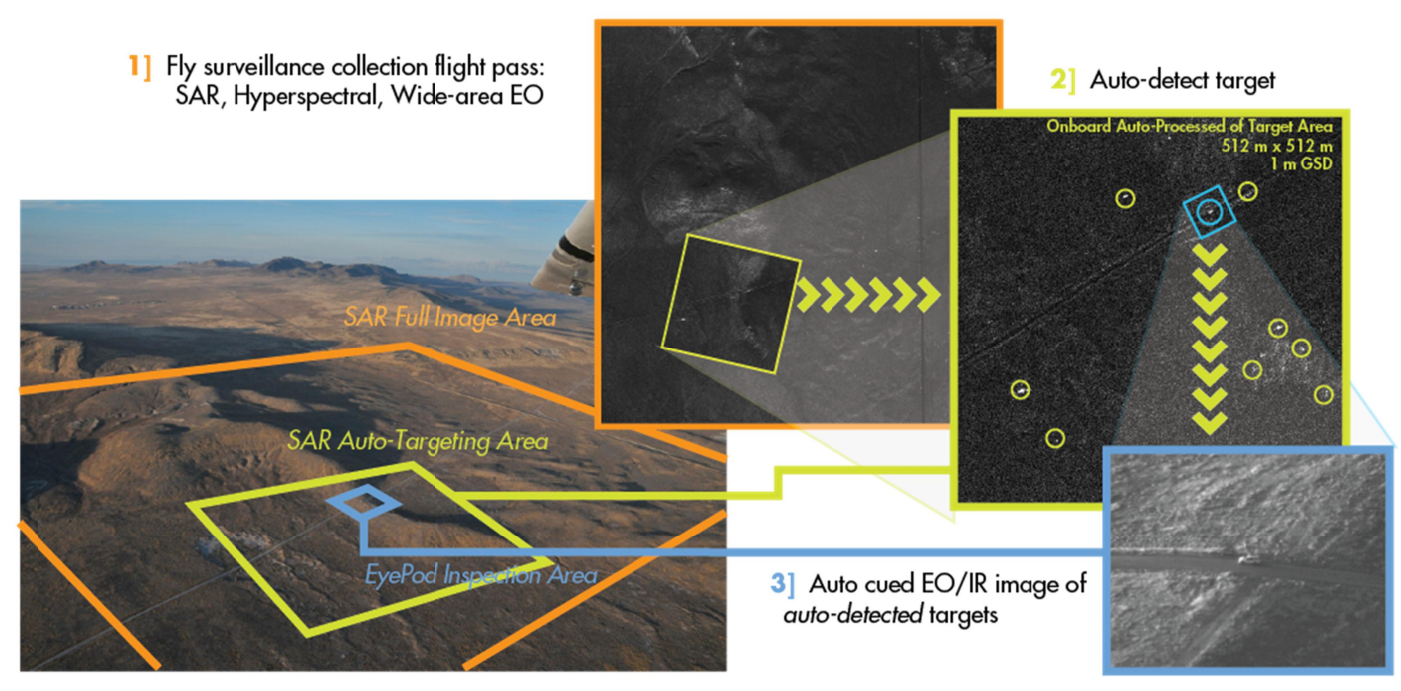

Figure 16. RASAR was used to cue EyePOD from auto-generated SAR images, which were scanned using CFAR algorithms to pull out detections.

\section{PATH FORWARD AND CONCLUSIONS}

We have presented the design and development of a modular, sensor management suite to enable real-time, scene-based collaboration between autonomous sensors and human operators. This central management functionality is the key to enabling tactical, mission-critical data fusion between disparate sensing modalities. The test and evaluation of the SMS system has focused on the tightly integrated sensor suite for the ONR TEISIT program. However, the modularity built into the architecture enables more flexible and advanced developments. Specifically, the existing SMS implementation can be extended to an enterprise level to include computing clouds and teaming of SMS systems on disjoint UASs. This enterprise solution would enable UAS teams, potentially carrying different sensor payloads or processing capabilities, to work together autonomously within a defined area of operation. Additional developments underway include the ability to leverage off-board data sources to intelligently control the on-board sensor suite and providing a means for warfighters to interact with the SMS system via text or voice commands.

Additionally, as autonomous flight algorithms and UAS auto-pilot technology improves, we envision an integrated system where the SMS and aircraft flight controls can work interactively to provide the end-user analyst the best possible ISR data for analysis and data fusion, by not only re-tasking the sensor suite, but re-tasking the UAS as well.

\section{REFERENCES}

[1] A. Bird, S. A. Anderson, D. Linne von Berg, M. Davidson, Niel Holt, M. Kruer, M. L. Wilson, "Compact Survey and Inspection Day/Night Image Sensor Suite for Small Unmanned Aircraft Systems (EyePod)," in Proc. SPIE 
7668, Airborne Intelligence, Surveillance, Reconnaissance (ISR) Systems and Applications VII, 766809 (April 24, 2010); doi:10.1117/12.850805.

[2] A. Bird, S. A. Anderson, D. Linne von Berg, N. Holt, M. Kruer, M. L. Wilson, "Multi-Sensor Airborne Imagery Collection and Processing Onboard Small Unmanned Systems," in Proc. Military Sensing Symposia (MSS) Passive Sensors, Orlando, FL, 2010.

[3] T. J. Walls, M. L. Wilson, C. Knight, D. Madsen, M. Jensen, S. A. Anderson, D. Partridge, M. Addario, D.Colclough, "Applications of Compact, Tactical Synthetic Aperture Radars," Proc. Military Sensing Symposia (MSS) Passive Sensors, Pasadena, CA, 2012.

[4] M. L. Wilson, D. Linne von Berg, M. Kruer, S. A. Anderson, N. Holt, M. Jensen, T. J. Walls, "Combined Survey and Inspection Optical and SAR Imaging for Small Airborne Platforms," Proc. Military Sensing Symposia (MSS) Passive Sensors, Orlando, FL, 2009. 